\title{
Introduction for solid state membranes for bio-molecules sensing and manipulation
}

\author{
Jean-Pierre Leburton · Massimiliano Di Ventra
}

Published online: 10 October 2014

(C) Springer Science+Business Media New York 2014

\section{Introduction}

Today's semiconductor nanotechnology has advanced to fabricate solid-state structures with fearure sizes comparable to the separation between atoms in bio-molecular systems. At the same time, progress in mesoscopic physics has demonstrated sensing capabilities of nanoscale capacitors corresponding to a fraction of the electron charge, which in principles enables probing single biomolecules electrically. In this context, the idea of engineering nanopores in ultra-thin solid-state membranes to mimic the operation of biological ion channels in living cells has emerged as a new way to investigate biological processes with semiconductor nanotechnology. Indeed, the versatility of semiconductor materials in terms of conduction polarity by selective $\mathrm{n}$ - or p-doping, as well as their ability to tailor space charge at the surface provide not only electrical detection sensitivity for biological objects, but also new means to shape the electrostatic landscape close to the membrane. Therefore the potential of semiconductor nanostructures to sense, read and manipulate biomolecules electronically opens up new scientific and technological avenues in traditional life science disciplines such as genomics, proteomics, etc.

These last years have experienced a considerable scientific interest in investigating the interaction between solid-state membranes and biological systems for potential applications in DNA/RNA sequencing. Indeed, if successful, this new approach based on semiconductor nanotechnology would offer tremendous advantages in terms of cost, speed and reli-

J.-P. Leburton $(\varangle) \cdot$ M. Di Ventra

University of Illinois, Urbana, IL, USA

e-mail: jleburto@illinois.edu

M. Di Ventra

University of California, San Diego, CA, USA ability over traditional bio-chemical techniques. In addition, the miniaturization capability of semiconductor materials will provide the natural electronic ingredients for new compact devices with a broad range of diagnostic or therapeutic applications in personal medicines. Realization and investigation of such bio-nano-electronic systems is not straightforward, neither from an experimental nor a theoretical viewpoint as one deals with a non-homogenous multi-phase material systems consisting of bio-molecular-electronic interaction at nanoscale solid-liquid interfaces. In this context, new methodologies merging biological techniques with solid sate physics and device engineering need to be developed. From a simulation point of view, this requires the integration of molecular dynamics that describe the behavior of biomolecules in solution with nanoscale device modeling tools that simulate the electrical response of the semiconductor membranes.

This special issue presents pioneering computational approaches to meet this challenge. In a scientific introduction to the scope of this special issue, A.A. Lucas at the University of Namur, Belgium provides an overview of DNA structures as the hardware and software of life. P. Boynton et al., at the University of California, San Diego present a new proposal for improving sequencing DNA by tunneling in solid-state membranes. C. Heitzinger at Arizona State University then discusses an approach based on a hierarchy of transport equations to model bio-nano-electronic interaction in nanopores. A.A. Aksimentiev at the University of Illinois at UrbanaChampaign presents his molecular dynamics approach to model thermophore in solid state nanopores. A. Girdhar et $a l$. also at UIUC describe an integrated molecular dynamics device modeling approach to simulate and optimize the sensitivity of the electronic response of a graphene membrane to DNA in the nanopore. B.K Nikolic and his group report on a comparison between first-principles analysis and semi- 
empirical modeling of transport in graphene sensors for DNA sequencing. Finally, M. Gracheva, at Clarkson University, NY describes a Poisson-Nernst-Planck model for an ionic transistor based on a semiconductor membrane.

This special issue of Journal of Computational Electronics devoted to the "Computational Approaches to Solid State Membranes for DNA sensing /sequencing" will definitely be required reading for those with interest in investigating this new realm of biology-nano-electronics interaction.

Jean-Pierre Leburton Guest Editor
Massimiliano Di Ventra Guest Editor 